\title{
Ações de alimentação e nutrição na atenção primária à saúde no Brasil
}

\author{
Gisele Ane Bortolini', Thais Fonseca Veloso de Oliveira1', Sara Araújo da Silva1, Rafaella da Costa \\ Santin ${ }^{1}$, Olivia Lucena de Medeiros ${ }^{1}$, Ana Maria Spaniol', Ana Carolina Lucena Pires ${ }^{1}$, Maria Fernanda \\ Moratori Alves e Lívia de Almeida Faller ${ }^{1}$
}

Como citar

Bortolini GA, Oliveira TFV, Silva SA, Santin RC, Medeiros OL, Spaniol AM, et al. Ações de alimentação e nutrição na atenção primária à saúde no Brasil. Rev Panam Salud Publica. 2020;44:e39. https://doi.org/10.26633/RPSP.2020.39

RESUMO

No Brasil, o Sistema Único de Saúde (SUS), público e universal, oferece ações de promoção, proteção e recuperação da saúde. A atenção primária à saúde (APS) é a porta preferencial de acesso dos indivíduos ao SUS e tem como papel coordenar e ordenar as ações e os serviços de saúde disponibilizados na rede. No âmbito da APS, as ações de alimentação e nutrição devem estar alinhadas às diretrizes da Política Nacional de Alimentação e Nutrição (PNAN) e podem ser potencializadas a partir de ações de vigilância alimentar e nutricional. Dados de sistemas de informação disponíveis em todas as unidades básicas de saúde e em inquéritos populacionais indicam que mais da metade da população adulta no Brasil apresenta excesso de peso e que o consumo de alimentos ultraprocessados vem aumentando. Essa situação exige que as equipes de saúde se organizem de forma a priorizar ações para indivíduos com agravos crônicos com base em estratificação de risco, estabilização da condição e potencialização do autocuidado apoiado, com foco em alimentação e atividade física. Ao mesmo tempo, dependendo do perfil epidemiológico, as equipes devem empreender ações de combate à desnutrição, prevenção da anemia e hipovitaminose $A$, considerando a múltipla carga da má nutrição no país. O presente artigo tem como objetivo apresentar o panorama atual das ações de alimentação e nutrição implementadas no âmbito da APS no SUS.

Palavras-chave Política pública; obesidade; diabetes mellitus; hipertensão; programas e políticas de nutrição e alimentação; Brasil.

A má alimentação é um dos principais fatores de risco relacionados à carga global de doenças no mundo. No Brasil, em 2015, ela foi o fator de risco que mais contribuiu para os anos de vida perdidos, sendo superior, inclusive, ao uso de álcool, drogas, tabagismo e inatividade física (1). Ainda, a alimentação inadequada foi o principal fator de risco para mortes no mundo em 2017 (2).

Como chamamento aos países para enfrentarem todas as formas de má nutrição, considerando sua multicausalidade - que engloba a desnutrição, as carências de micronutrientes, o sobrepeso e a obesidade - a Organização das Nações Unidas para a
Alimentação e a Agricultura (FAO) e a Organização Mundial da Saúde (OMS) lançaram, para o período de 2016 a 2025, a Década de Ação das Nações Unidas para a Nutrição (3). O Brasil foi protagonista nessas discussões, sendo o primeiro país a firmar compromissos no contexto da Década. Atualmente, o Brasil coordena duas Redes de Ação para países da América Latina e Caribe: uma sobre guias alimentares baseados em alimentos (ao invés de baseados em nutrientes) e outra sobre redução do consumo de sal para prevenção de doenças cardiovasculares.

A alimentação foi reconhecida como direito na Constituição brasileira em 2010 (4) e como um dos determinantes da saúde

\footnotetext{
1 Ministério da Saúde, Secretaria de Atenção Primária à Saúde, Departamento de Promoção da Saúde, Coordenação-Geral de Alimentação e Nutrição, Brasília (DF), Brasil. $\triangle$ Gisele Ane Bortolini, gisele.bortolini@saude.gov.br
} 
da população na lei que criou o Sistema Único de Saúde (SUS); cabe à direção nacional do SUS a competência de "formular, avaliar e apoiar políticas de alimentação e nutrição" (5). As ações de alimentação e nutrição no SUS foram institucionalizadas a partir da publicação da Política Nacional de Alimentação e Nutrição (PNAN), em 1999 (6). Em 2011, uma atualização da PNAN agregou a essa política o propósito de melhorar as condições de alimentação, nutrição e saúde da população brasileira pela promoção de práticas alimentares adequadas e saudáveis, vigilância alimentar e nutricional (VAN), prevenção e cuidado integral dos agravos relacionados à alimentação e nutrição (7). Atualmente, o cenário epidemiológico no Brasil mostra uma situação nutricional bastante complexa, cujo enfrentamento depende de um amplo leque de ações. Pesquisas e inquéritos populacionais mostram que, no país, $55,7 \%$ das pessoas maiores de 18 anos apresentam excesso de peso; a obesidade afeta 19,8\%, a hipertensão, 24,7\% e o diabetes, 7,7\% (8). De 2006 a 2018 , as prevalências de excesso de peso, obesidade e diabetes tiveram incremento de $30 \%, 65 \%$ e $40 \%$, respectivamente, enquanto a prevalência de hipertensão não apresentou uma variação anual estatisticamente significativa (8).

Em crianças, 18,8\% das menores de 2 anos, 14,3\% daquelas com idade de 2 a 4 anos e $24,4 \%$ das crianças de 5 a 10 anos apresentam excesso de peso (9). Entre jovens de 18 a 24 anos, o excesso de peso cresceu $56 \%$ e a obesidade, $110 \%$ no período de 2006 a 2017 (10). Destaca-se que o peso corporal é o principal motivo para a busca de serviços de saúde por adolescentes (11).

Por sua vez, a desnutrição em nível nacional diminuiu nas últimas décadas. Em 2006, aproximadamente 7\% das crianças menores de 5 anos apresentavam déficit de crescimento e 1,5\% apresentava déficits de peso em relação à altura. Nesse mesmo público, 20,9\% apresentavam anemia e 17\% apresentavam hipovitaminose A (12). A desnutrição permanece como problema de saúde importante no país, principalmente nas crianças menores de 5 anos pertencentes a povos e comunidades tradicionais. Em 2016, 8\% das crianças indígenas e 6,1\% das quilombolas apresentaram baixo peso. A deficiência de estatura para a idade foi identificada em $30,5 \%$ das indígenas e $17,0 \%$ das quilombolas nesse mesmo ano (13).

Os compromissos assumidos no âmbito da Década de Ação das Nações Unidas para a Nutrição dialogam com as diretrizes da PNAN, que indicam as linhas de ação para melhoria das condições de alimentação, nutrição e saúde da população brasileira. Considerando que os serviços de saúde situados no ambiente onde as pessoas vivem desempenham um papel central na promoção da saúde, prevenção e cuidado, o objetivo deste artigo é apresentar o panorama atual das ações de alimentação e nutrição implementadas no âmbito da atenção primária à saúde (APS) no SUS.

\section{ATENÇÃO NUTRICIONAL NO SUS: CONTEXTUALIZAÇÃO}

A atenção nutricional, primeira diretriz da PNAN, compreende os cuidados relativos a alimentação e nutrição voltados à promoção e proteção da saúde, prevenção, diagnóstico e tratamento de agravos, que devem estar associados às demais ações de atenção à saúde do SUS para indivíduos, famílias e comunidades. A atenção nutricional deve dialogar com as demandas e necessidades de saúde do território, considerando as de maior frequência e relevância e observando critérios de risco e vulnerabilidade, e deve ainda fazer parte do cuidado integral na rede de atenção à saúde (RAS) (7).

O primeiro nível de assistência do sistema de saúde, a APS, se caracteriza pela longitudinalidade e integralidade nas ações e pela coordenação do cuidado a partir de uma perspectiva de integração dos serviços de saúde em determinado território. A APS deve ser acessível a todos e oferecer os serviços essenciais para prevenir e tratar doenças, sendo responsável ainda pela promoção da saúde, reabilitação e cuidados paliativos (14). Como a APS é responsável pela resposta à maior parte dos problemas de saúde das pessoas, seu funcionamento é crítico para o sistema de saúde, especialmente em contextos de envelhecimento e adoecimento da população (15).

No Brasil, a consolidação da APS tem como modelo prioritário a Estratégia Saúde da Família (ESF) (16), operacionalizada por equipes multiprofissionais que implementam os programas e estratégias da Política Nacional de Atenção Básica (PNAB), que são, por sua vez, monitorados por meio do Sistema de informação em Saúde para a Atenção Básica (SISAB). Evidências demonstram os impactos positivos da expansão da cobertura da ESF na saúde da população brasileira: a ESF está associada à redução da mortalidade infantil e da mortalidade por infecções respiratórias e diarreia em menores de 5 anos (17), à diminuição das hospitalizações por doenças potencialmente evitáveis (18) e à redução da mortalidade por doenças cerebrovasculares e cardiovasculares (19). O número de equipes da ESF cresceu rapidamente ao longo das últimas décadas, principalmente nos municípios de menor porte populacional. Atualmente, há 43332 equipes de ESF em 5483 (98\%) municípios, atendendo mais de 133 milhões de brasileiros, ou seja, 63\% da população do país.

\section{PRINCIPAIS AÇÕES DE ALIMENTAÇÃO E NUTRIÇÃO NA APS}

\section{Vigilância alimentar e nutricional}

Define-se VAN como a descrição contínua e a predição de tendências das condições de alimentação e nutrição da população e de seus fatores determinantes para subsidiar o planejamento de ações para prevenção e enfrentamento dos agravos relacionados à alimentação e nutrição, contribuindo com a organização da atenção nutricional na RAS. A VAN compreende desde os inquéritos populacionais até a avaliação das condições de alimentação e nutrição nos serviços de saúde, especialmente na $\operatorname{APS}(7,20)$. Embora a alimentação inadequada seja o fator de risco que mais impacta na carga de doença no Brasil, e apesar de mais de $50 \%$ dos adultos brasileiros apresentarem excesso de peso, a incorporação da avaliação do estado nutricional e do consumo alimentar na rotina das equipes da APS ainda é um grande desafio.

No âmbito da VAN, cabe aos profissionais da APS avaliar o estado nutricional (peso, altura e outros indicadores) e o consumo alimentar por meio de marcadores de consumo (20). Para registro das informações, foi disponibilizado a todos os municípios brasileiros o Sistema de Vigilância Alimentar e Nutricional (SISVAN). A partir dos dados coletivos, as equipes de saúde e os gestores municipais, estaduais e federais podem monitorar o padrão alimentar e o estado nutricional de sua população, organizar ações para prevenção e controle dos principais agravos relacionados a alimentação e nutrição em seu território e ofertar 
TABELA 1. Estado nutricional e marcadores de consumo alimentar para a população adulta e idosa atendida na atenção primária à saúde no Brasil, 2018

\begin{tabular}{|c|c|c|c|c|}
\hline \multirow[t]{2}{*}{ Variável analisada } & \multicolumn{2}{|c|}{ Adultos $^{\mathrm{a}}$} & \multicolumn{2}{|c|}{ Idosos $^{\mathrm{b}}$} \\
\hline & No. absoluto & $\%(I C 95 \%)$ & No. absoluto & $\%(1 \mathrm{C} 95 \%)$ \\
\hline \multicolumn{5}{|l|}{ Estado nutricional de acordo com índice de massa corporal ${ }^{\complement}$} \\
\hline Baixo peso & 346257 & $2,42(2,41$ a 2,43$)$ & 295494 & $12,46(12,41$ a 12,50$)$ \\
\hline Eutrofia & 5092961 & $35,61(35,59$ a 35,64$)$ & 868388 & $36,61(36,55$ a 36,67$)$ \\
\hline Sobrepeso & 4967566 & $34,74(34,71$ a 34,76$)$ & 1208287 & $50,94(50,87$ a 51$)$ \\
\hline Obesidade & 3893299 & $27,23(27,20$ a 27,25$)$ & - & - \\
\hline \multicolumn{5}{|l|}{ Marcadores de consumo alimentar ${ }^{d}$} \\
\hline Consumo de feijão & 548396 & $85,30(85,21$ a 85,39$)$ & 189421 & $86,28(86,14$ a 86,42$)$ \\
\hline Consumo de fruta & 473,283 & $73,62(73,51$ a 73,72$)$ & 170517 & $77,67(77,50$ a 77,84$)$ \\
\hline Consumo de verduras e legumes & 495472 & $77,07(76,97$ a 77,17$)$ & 178155 & $81,15(80,99$ a 81,31$)$ \\
\hline Consumo de hambúrguer e/ou embutidos & 233296 & $36,29(36,17$ a 36,41$)$ & 60756 & $27,67(27,49$ a 27,86$)$ \\
\hline Consumo de bebidas adoçadas & 348432 & $54,20(54,08$ a 54,32$)$ & 90214 & $41,09(40,89$ a 41,30$)$ \\
\hline Consumo de macarrão instantâneo, salgadinho de pacote ou biscoito salgado & 204044 & $31,74(31,62$ a 31,85$)$ & 53352 & $24,30(24,12$ a 24,48$)$ \\
\hline Consumo de biscoito recheado, doces ou guloseimas & 222272 & $34,57(34,46$ a 34,69$)$ & 52637 & $23,98(23,80$ a 24,16$)$ \\
\hline
\end{tabular}

ações de acordo com a necessidade das pessoas em todas as fases do curso da vida (20).

Em 2018, foram registrados no SISVAN o peso e a estatura de mais de 40 milhões de brasileiros de todas as faixas etárias, o que corresponde a uma cobertura populacional de 20,7\%. Desses, $62 \%$ dos adultos e $50,9 \%$ dos idosos apresentaram excesso de peso $(21,22)$. Nesse mesmo ano, cerca de 1,6 milhão de pessoas tiveram seu consumo alimentar avaliado, com cerca de $85,3 \%$ dos adultos e $86,3 \%$ dos idosos referindo consumo de feijão no dia anterior e $54,2 \%$ dos adultos e $41,1 \%$ dos idosos declarando consumo de bebidas adoçadas (tabela 1).

Além dos dados inseridos no próprio SISVAN, esse sistema também reúne e consolida os registros provenientes do SISAB e do acompanhamento do estado nutricional dos beneficiários do Programa Bolsa Família (20). No primeiro semestre de 2019, 4,6 milhões de crianças e cerca de 329 mil gestantes tiveram seu estado nutricional avaliado por uma equipe da APS a fim de cumprir uma das condicionalidades de saúde para recebimento do benefício (23).

\section{Promoção da alimentação adequada e saudável}

A promoção da alimentação adequada e saudável é compreendida como um conjunto de estratégias que proporcionam aos indivíduos e coletividades a realização de práticas alimentares apropriadas aos seus aspectos biológicos e socioculturais, bem como ao uso sustentável do ambiente (7). Dados do SISVAN em relação ao consumo alimentar apontam que, no Brasil, 54,4\% das crianças menores de 6 meses são amamentadas exclusivamente, 51,6\% são amamentadas na idade de 6 a 24 meses e $49 \%$ das crianças de 6 a 23 meses consomem alimentos ultraprocessados. Entre crianças de 5 a 10 anos, 68\% consomem bebidas adoçadas e $62 \%$ consomem macarrão instantâneo, salgadinhos de pacote ou biscoitos salgados (9). Em adultos, observa-se a redução do consumo de alimentos in natura e minimamente processados, como arroz e feijão, e o aumento do consumo de alimentos ultraprocessados (24).
Diante desse cenário, cabe às equipes da APS a realização de ações educativas e aconselhamento nas consultas individuais ou em grupos, apoiadas nas diretrizes oficiais para alimentação adequada e saudável apresentadas no Guia Alimentar para Crianças Brasileiras Menores de Dois Anos (25) e no Guia Alimentar para a População Brasileira (26), cuja implementação traz benefícios já evidenciados $(27,28)$. No entanto, o reconhecimento, pelas equipes da APS, das potencialidades e dificuldades do território em relação à alimentação saudável permanece como um desafio. Para desenvolver ações de promoção da saúde é importante identificar no território locais de produção, comercialização e distribuição de alimentos, assim como costumes e tradições alimentares locais que possam relacionar-se aos hábitos alimentares e ao estado nutricional da população. Com base na realidade local observada, as equipes podem incentivar e apoiar a criação de ambientes promotores de alimentação adequada e saudável, incidindo sobre a oferta de alimentos saudáveis nas escolas, nos ambientes de trabalho e no comércio local, além de articular com a agricultura local a criação de hortas comunitárias.

Para qualificar as ações de promoção do aleitamento materno e alimentação complementar no âmbito da APS, desde 2013 o Ministério da Saúde implementa a Estratégia Amamenta e Alimenta Brasil (EAAB) (29). Até o momento já foram formados 5959 tutores, que realizaram, por sua vez, oficinas de trabalho em 3290 Unidades Básicas de Saúde (UBS), qualificando 48640 profissionais da APS; 192 UBS já foram certificadas. As avaliações apontam para a melhoria nas prevalências de aleitamento materno e alimentação complementar no território das equipes envolvidas na estratégia $(30,31)$. As equipes da APS também devem se envolver com as escolas do seu território por meio do Programa Saúde na Escola (PSE), no qual desenvolvem ações conjuntas de promoção da saúde, como, por exemplo, a promoção da alimentação saudável (32).

Os municípios que aderem ao PSE também podem receber um incentivo extra do Ministério da Saúde por meio da adesão ao Programa Crescer Saudável, cujo objetivo é prevenir e 
controlar a obesidade infantil. No ciclo atual (2019/2020), aderiram ao Crescer Saudável 4118 municípios, sendo liberados R\$ 38,8 milhões para o cumprimento de metas relacionadas à avaliação do estado nutricional das crianças, realização de ações de promoção da alimentação saudável e de práticas corporais e atividade física e oferta de cuidados nas UBS para as crianças identificadas com obesidade (33).

As equipes da APS devem promover a alimentação adequada e saudável e a prática de atividades físicas considerando as especificidades, desejos e possibilidades de cada indivíduo. Da mesma forma, devem identificar parceiros e recursos no território para o desenvolvimento de ações intersetoriais que possam estimular a criação de ambientes propícios para a adoção de estilos de vida mais saudáveis por parte da população. Já que a escola é um espaço propício para a formação e a discussão de práticas saudáveis, a ação conjunta com as escolas é fundamental para combater e prevenir a obesidade infantil e em adolescentes.

A promoção da alimentação saudável na APS passa pela apropriação, por parte dos profissionais de saúde, das orientações dos guias alimentares, especialmente no que diz respeito aos efeitos prejudiciais do consumo de alimentos ultraprocessados. Considerando o aumento da prevalência de obesidade infantil no país (9), atenção especial deve ser dada às ações de promoção do aleitamento materno e introdução da alimentação complementar.

\section{Prevenção das carências nutricionais}

Embora o sobrepeso e a obesidade estejam hoje entre os principais desafios do Brasil relacionados à alimentação e nutrição, ainda persistem carências nutricionais (anemia e hipovitaminose A) combatidas por meio da suplementação de micronutrientes. Cabe às equipes da APS ofertar suplementos de ferro e vitamina A às crianças que frequentam as UBS por meio dos dois principais programas de suplementação do país: Programa Nacional de Suplementação de Ferro e Programa Nacional de Suplementação de Vitamina A.

As crianças que frequentam as UBS recebem suplementos de ferro na idade de 6 a 24 meses em todos os municípios brasileiros. Já os suplementos de vitamina A são distribuídos nas UBS de todos os municípios da região Norte e Nordeste e em alguns municípios das regiões Sudeste, Centro-Oeste e Sul que apresentam maiores prevalências da deficiência, totalizando 3546 municípios participantes do programa. Desde 2005, anualmente cerca de 3,7 milhões de crianças de 6 a 59 meses de idade recebem suplementos nos municípios e Distritos Sanitários Especiais Indígenas.

Em outra ação mais recente, que acontece desde 2014, crianças de 6 a 48 meses que frequentam creches aderidas ao PSE recebem o NutriSUS - que consiste na adição de um suplemento contendo 15 vitaminas e minerais em pó à comida ofertada às crianças. A ação foi testada e ampliada para todo o país após resultados positivos em estudo multicêntrico realizado em cinco cidades brasileiras (34). No primeiro semestre de 2019, quase 215 mil crianças em 6319 creches e 20 Distritos Sanitários Especiais Indígenas receberam o suplemento.

Atualmente, o Ministério da Saúde financia um Inquérito Nacional que permitirá, em 2020/2021, rediscutir a agenda de prevenção e controle das carências nutricionais no país, visto que os últimos dados sobre a prevalência de carências nutricionais entre a população brasileira são de 2006 (12).

\section{Manejo dietético da obesidade, diabetes e hipertensão arterial sistêmica}

Para o cuidado dos usuários com obesidade, diabetes e hipertensão, as equipes da APS ofertam, entre outros, consultas individuais, atividades em grupos e visitas domiciliares. Em 2013, o Ministério da Saúde redefiniu diretrizes para organização da RAS das pessoas com doenças crônicas não transmissíveis (DCNT) no âmbito do SUS, com objetivo de organizar a atenção integral à saúde de maneira articulada entre os diferentes níveis de atenção do SUS.

Para organização das ofertas de cuidado para pessoas com obesidade, municípios, regiões de saúde e estados se organizam localmente na implementação da Linha de Cuidado de Sobrepeso e Obesidade (35), que organiza o percurso dos indivíduos dentro do sistema de acordo com a gravidade da condição. Até dezembro de 2019, 21 unidades federadas brasileiras apresentaram processos em fase de implantação das LCSO, totalizando 43 linhas em todo o país.

A obesidade é o terceiro principal fator de risco que provoca a maioria das mortes e incapacidades em mulheres no Brasil, e o quinto entre homens (1). As atribuições da APS no cuidado à pessoa com obesidade envolvem VAN, estratificação de risco, ações de promoção da saúde intersetoriais e com participação social, promoção da alimentação adequada e saudável, apoio ao autocuidado, assistência terapêutica multiprofissional, inclusive àqueles que realizaram procedimento cirúrgico, coordenação do cuidado (encaminhamento aos outros níveis de atenção) e acolhimento adequado (35).

De acordo com dados do SISAB, é possível observar que, no período de setembro de 2018 a agosto de 2019, dos mais de 105 milhões de atendimentos realizados e passíveis de identificação nesse sistema, 26,38\% das condições/problemas avaliados correspondiam à hipertensão arterial, 10,51\% ao diabetes e apenas $2,85 \%$ correspondiam à obesidade (dados não apresentados em tabela).

A tabela 2 apresenta as características das condições avaliadas com relação à conduta/desfecho segundo ficha de atendimento individual do SISAB no período de setembro de 2018 a agosto de 2019. Como mostra a tabela 2 , dos casos de obesidade cuja conduta/desfecho resultou na permanência do acompanhamento no âmbito da APS, 46,70\% foram agendados para cuidado continuado/programado (conduta aplicada para casos de DCNT) e $41,54 \%$ para consulta agendada. Observou-se que $22,48 \%$ dos casos tiveram alta do episódio, o que indica que não houve cuidado continuado, apesar da condição de obesidade avaliada. Dentre os casos encaminhados para outros pontos da RAS, 94,97\% tiveram encaminhamento para serviço especializado.

No Brasil, entre as pessoas com hipertensão, 36,7\% têm obesidade e $74,4 \%$ têm excesso de peso; entre aqueles com diabetes, $75,2 \%$ têm excesso de peso e $37 \%$ têm obesidade; e entre pessoas com hipertensão e diabetes, $80,1 \%$ têm excesso de peso (36). Considerando que um quarto das condições ou problemas avaliados no SISAB correspondiam à hipertensão arterial e 10,51\% ao diabetes, podemos concluir que a obesidade ainda é uma condição invisibilizada no âmbito da APS, com o desafio de ser identificada como problema pelos profissionais de saúde para receber o acompanhamento adequado por meio de cuidado continuado, como outras DCNT.

Outra ação importante das equipes de APS é a realização de atividades coletivas. Segundo dados do SISAB, no período de 


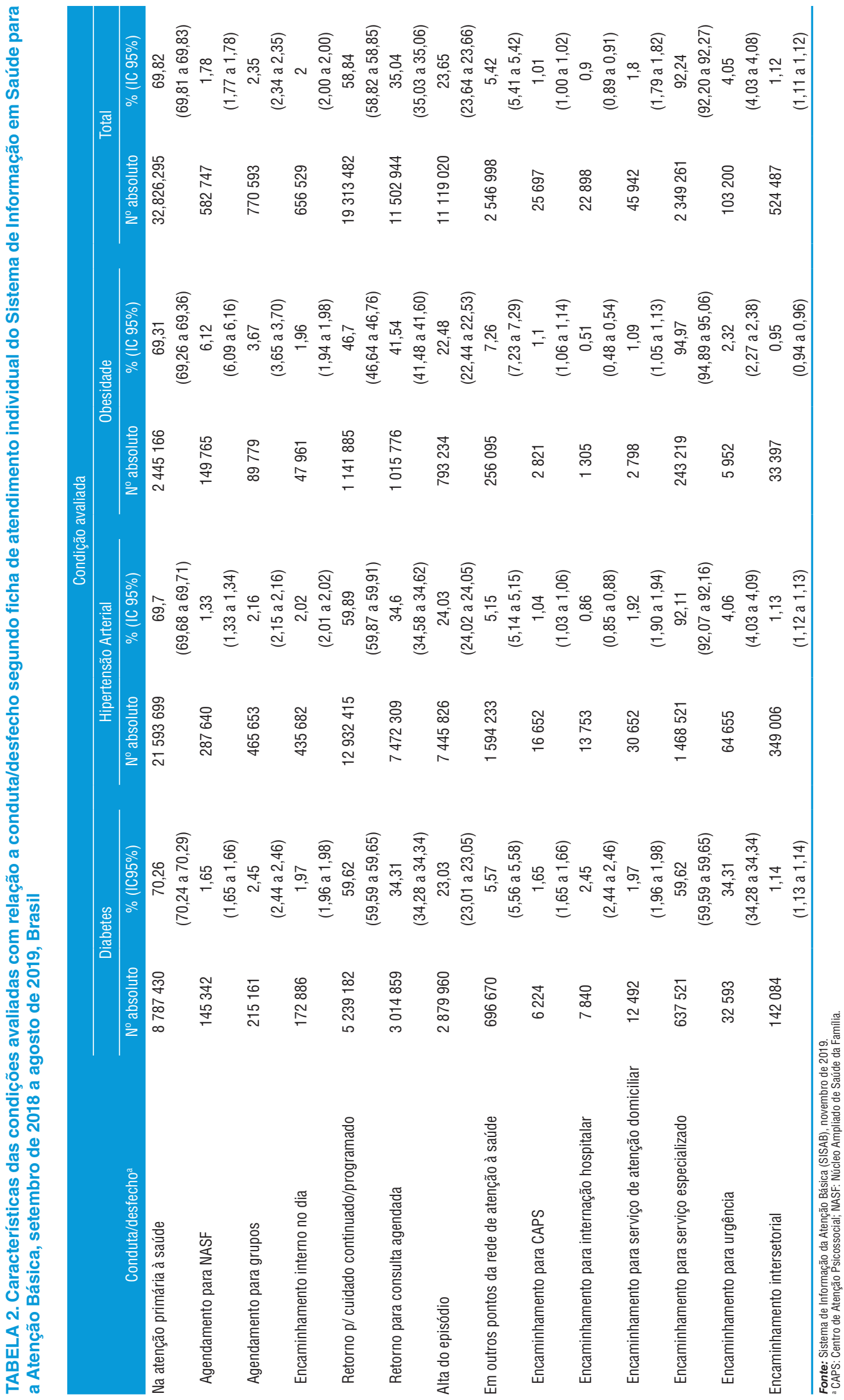


setembro de 2018 a agosto de 2019, mais de 807 mil atividades coletivas foram registradas pelas equipes. Dentre os temas abordados, 20,29\% foram relacionados à alimentação saudável e $48,30 \%$ ao autocuidado de pessoas com DCNT. Essas atividades envolveram 1773219 e 4104816 participantes, respectivamente. Dentre as práticas de saúde realizadas nas atividades coletivas, 12,29\% estavam relacionadas a avaliação antropométrica e $76,62 \%$ a práticas corporais e atividade física. Tais práticas envolveram 650024 e 3638857 participantes, respectivamente.

Observa-se que, apesar de um quinto das ações coletivas abordarem alimentação saudável, quase metade abordar o autocuidado de pessoas com DCNT e mais de dois terços envolverem práticas corporais e atividade física - ações que podem contar com a participação das pessoas com obesidade ou serem voltadas a essas pessoas -, a inserção desses usuários na rotina das equipes da APS parece estar mais restrita ao âmbito coletivo do que individual, tendo em vista que o percentual de consultas não chega nem a $3 \%$ do total.

Para apoiar as equipes no cuidado, são disponibilizadas diretrizes e estratégias para atenção às pessoas com doença crônica, hipertensão arterial sistêmica, diabetes e obesidade. Além disso, são disponibilizados cursos de educação a distância por meio das plataformas UnaSUS (https://www. unasus.gov.br/), RedeNutri (http://ecos-redenutri.bvs.br/ tiki-view_articles.php), Telessaúde (https://www.saude.gov. br/telessaude/nucleos-de-telessaude) e Secretaria de Atenção Primária à Saúde (https://aps.saude.gov.br/) nas temáticas de alimentação adequada e saudável e obesidade. Atualmente, estão em elaboração um protocolo clínico para manejo da obesidade, um manual para a abordagem coletiva da obesidade na APS e um manual para tratamento da obesidade infantil e suas comorbidades.

No âmbito do cuidado, apesar das diretrizes e orientações publicadas pelo Ministério da Saúde sobre a atenção às pessoas com sobrepeso e obesidade, ainda é necessário avançar na incorporação dessas informações na rotina e nas práticas dos profissionais de saúde, por meio de uma abordagem ampla que envolva desde o diagnóstico nutricional até o desenvolvimento de ações de promoção da alimentação adequada e saudável e de atividades físicas.

É preciso intensificar ações de prevenção e promoção para que indivíduos com excesso de peso possam reduzir a gravidade dessa condição e, com isso, reduzir o risco de desenvolverem DCNT. Em relação à obesidade, é necessário que essa condição passe a ser considerada não apenas como uma doença importante, mas também como um grave fator de risco para outras DCNT, que merece atenção por parte dos profissionais de saúde e da população. Estudos apontam que, quando os profissionais de saúde não falam sobre a obesidade, os indivíduos presumem que ela não é um problema (37). Assim, o alto percentual de encaminhamento de usuários com obesidade para a atenção especializada indica que o tratamento e cuidado dessas pessoas na APS ainda é um desafio.

Destaca-se ainda a necessidade de qualificação dos profissionais da APS, em especial médicos e enfermeiros, para a oferta de orientações sobre alimentação adequada e saudável e sua sensibilização para o acolhimento das pessoas com obesidade. Dentre as maiores barreiras para a procura do serviço de saúde pelos usuários para tratamento da obesidade está o estigma e o preconceito vivenciado por essas pessoas. Contribui para isso a falta de adequação da infraestrutura dos serviços para o atendimento dessas pessoas (37).

\section{CONCLUSÕES}

No Brasil, a saúde e a alimentação são direitos fundamentais garantidos por meio da Constituição. As ações de alimentação e nutrição no Brasil iniciaram antes do SUS e se consolidam a partir do SUS, que já completou 30 anos de existência. Uma das ações mais consistentes na APS é a VAN, que permite conhecer o estado nutricional e o consumo alimentar da população ao longo das décadas e direciona o trabalho das equipes locais.

Outra iniciativa marcante na APS é a promoção da alimentação adequada e saudável, com ações realizadas, em sua maioria, em grupos ou em consultas individuais ou visitas domiciliares. Historicamente, as ações de promoção do aleitamento materno são as mais consolidadas, porém, nas últimas duas décadas, com a publicação de guias alimentares pelo Ministério da Saúde, outras temáticas passaram a fazer parte da abordagem das equipes. Também merecem destaque as ações intersetoriais que acontecem localmente para promoção saúde a partir das equipes de APS, como, por exemplo, o PSE, presente em quase todos municípios brasileiros.

Em relação aos agravos relacionados à alimentação inadequada, o excesso de peso é o principal agravo nutricional que acomete crianças, adolescentes, adultos e idosos no Brasil. O excesso de peso e a obesidade, sua forma mais grave, podem e devem ser prioridade na agenda das equipes de APS, e muitas vezes ocorrem de forma concomitante a agravos atualmente de menor magnitude, como a desnutrição e as deficiências de micronutrientes, que também são responsabilidade das equipes de APS. Em relação à obesidade, ainda existe a necessidade de avançar na identificação e intervenção precoce, anterior ao diagnóstico de hipertensão e diabetes.

Em síntese, é possível afirmar que a organização da atenção nutricional em sistemas de saúde universais deve iniciar pelo reconhecimento dos principais agravos relacionados à alimentação e à nutrição que acontecem no território das equipes de APS. Esse reconhecimento é facilitado quando são disponibilizados instrumentos e sistemas para registro e consolidação das informações, possibilitando um diagnóstico das necessidades e demandas da população adstrita. Com base no diagnóstico local, as equipes podem organizar ações de promoção, proteção e apoio, setoriais e intersetoriais, que contribuam para ampliar as formas de acesso e consumo de uma alimentação mais saudável. Tais ações também podem ser facilitadas com indução nacional por meio de financiamento e publicação de diretrizes oficiais.

São reconhecidos e inquestionáveis os ganhos em saúde para todos os brasileiros pela adoção, no país, de um sistema de saúde público e universal, que define que saúde é um direito de todos e um dever do Estado. Considerando que a alimentação inadequada e o excesso de peso lideram os fatores de risco que mais contribuem para a carga da doença dos brasileiros, os desafios da agenda de alimentação e nutrição APS no Brasil estão relacionados à ampliação do acesso e ao cuidado longitudinal e integral para um número cada vez maior de brasileiros.

Contribuição das autoras. SAS, RCS e AMS coletaram e analisaram os dados, e construíram as tabelas. GAB, TFVO, OLM, ACLP, MFMA e LAF interpretaram e escreveram o artigo. GAB 
e TFVO revisaram o artigo. Todas as autoras revisaram e aprovaram a versão final.

Conflitos de interesse. Nada declarado pelas autoras.
Declaração. As opiniões expressas no manuscrito são de responsabilidade exclusiva dos autores e não refletem necessariamente a opinião ou política da RPSP/PAJPH ou da Organização Pan-Americana da Saúde (OPAS).

\section{REFERÊNCIAS}

1. Malta DC, Felisbino-Mendes MS, Machado ÍE, De Azeredo Passos VM, De Abreu DMX, Ishitani LH, et al. Risk factors related to the global burden of disease in Brazil and its federated units, 2015. Rev Bras Epidemiol. 2017;20:217-32.

2. Stanaway JD, Afshin A, Gakidou E, Lim SS, Abate D, Abate KH, et al. Global, regional, and national comparative risk assessment of 84 behavioural, environmental and occupational, and metabolic risks or clusters of risks for 195 countries and territories, 1990-2017: A systematic analysis for the Global Burden of Disease Study 2017. Lancet. 2018 Nov 10;392(10159):1923-94.

3. World Health Organization (WHO), Food and Agriculture Organization of the United Nations. Driving commitment for nutrition within the UN Decade of Action on Nutrition: policy brief. Genebra: WHO; 2018. (WHO/NMH/NHD/17.11).

4. Brasil. Emenda Constitucional $n^{\circ}$ 64, de 4 de fevereiro de 2010. Altera o art. $6^{\circ}$ da Constituição Federal, para introduzir a alimentação como direito social. [Internet]. 2010. Available from: http:// www.planalto.gov.br/ccivil_03/constituicao/emendas/emc/ emc64.htm

5. Brasil. Lei No 8.080, de 19 de setembro de 1990. Dispõe sobre as condições para promoção, proteção e recuperação da saúde, a organização e o funcionamento dos serviços correspondentes e dá outras providências. 1990.

6. Brasil. Ministério da Saúde (MS). Secretaria de Atenção à Saúde. Departamento de Atenção Básica. Política Nacional de Alimentação e Nutrição [Internet]. Brasília: Ministério da Saúde; 2007 [cited 2020 Jan 22]. 48 p. Available from: www.saude.gov.br/bvs

7. Brasil. Ministério da Saúde. Secretaria de Atenção à Saúde. Departamento de Atenção Básica. Política Nacional de Alimentação e Nutrição. 1st ed. Brasília: Ministério da Saúde; 2013. 84 p.

8. Brasil. Ministério Da Saúde. Secretaria de Vigilancia em Saúde. Vigitel Brasil 2018: vigilância de fatores de risco e proteção para doenças crônicas por inquérito telefônico: estimativas sobre frequência e distribuição sociodemográfica de fatores de risco e proteção para doenças crônicas nas capitais dos 26 estados br [Internet]. Vigitel. 2019. 152 p. Available from: http://portal.saude.gov.br/portal/ arquivos/pdf/vigitel_2010_preliminar_web.pdf

9. Brasil. Ministério Da Saúde. DATASUS. Sistema de Vigilância Alimentar e Nutricional [Internet]. 2018 [cited 2019 Nov 1]. Available from: http:/ /sisaps.saude.gov.br/sisvan/relatoriopublico/index

10. Brasil. Ministério Da Saúde. Secretaria de Vigilancia em Saúde. Vigitel Brasil 2017: vigilância de fatores de risco e proteção para doenças crônicas por inquérito telefônico : estimativas sobre frequência e distribuição sociodemográfica de fatores de risco e proteção para doenças crônicas nas capitais dos 26 estados b [Internet]. Vigitel. 2018. 152 p. Available from: http://portal.saude.gov.br/portal/ arquivos/pdf/vigitel_2010_preliminar_web.pdf

11. Brasil. Ministério Da Saúde. Secretaria de Vigilância em Saúde. Uso de serviços de saúde e fatores associados à procura pela Unidade Básica de Saúde entre adolescentes brasileiros: resultados da Pesquisa Nacional de Saúde do Escolar (PeNSE), 2015. 2019.

12. Brasil. Ministério da Saúde. Pesquisa Nacional de Demografia e Saúde da Criança e da Mulher - PNDS 2006 : dimensões do processo reprodutivo e da saúde da criança. Brasília; 2009.

13. Brasil. Câmara Interministerial de Segurança Alimentar e Nutricional. Mapeamento da Insegurança Alimentar e Nutricional com foco na Desnutrição a partir da análise do Cadastro Único, do Sistema Nacional de Vigilância Alimentar e Nutricional (SISVAN) e do Sistema de Informação da Atenção à Saúde Indígena (SIASI) 2016 [Internet]. Brasília; 2018 [cited 2020 Jan 22]. Available from: http://www.mds.gov.br/webarquivos/arquivo/ seguranca_alimentar/caisan/Publicacao/Caisan_Nacional/ Estudo_tecnico_MapaInsan_2018.pdf
14. World Health Organization. Declaration of Astana. Global Conference on Primary Health Care [Internet]. 2018 [cited 2019 Nov 3]. Available from: https://www.who.int/docs/default-source/primary-health/ declaration/gcphc-declaration.pdf

15. Starfield B, Shi L, Macinko J. Contribution of primary care to health systems and health. Vol. 83, Milbank Quarterly. 2005. p. 457-502.

16. Ministério da Saúde. Portaria de Consolidação n ${ }^{\circ}$ 02, Anexo XXII, de 28 de setembro de 2017. Aprova a Política Nacional de Atenção Básica. 2017.

17. Rasella D, Aquino R, Barreto ML. Reducing childhood mortality from diarrhea and lower respiratory tract infections in brazil. Pediatrics. 2010 Sep;126(3).

18. Macinko J, Starfield B, Shi L. The contribution of primary care systems to health outcomes within Organization for Economic Cooperation and Development (OECD) countries, 1970-1998. Health Serv Res. 2003 Jun;38(3):831-65.

19. Rasella D, Harhay MO, Pamponet ML, Aquino R, Barreto ML. Impact of primary health care on mortality from heart and cerebrovascular diseases in Brazil: A nationwide analysis of longitudinal data. BMJ. 2014 Jul 3;349.

20. Brasil. Ministério da Saúde. Secretaria de Atenção à Saúde. Departamento de Atenção Básica. Marco de Referência da Vigilância Alimentar Nutricional [Internet]. 2015 [cited 2020 Jan 22]. Available from: http://bvsms.saude.gov.br/bvs/publicacoes/ marco_referencia_vigilancia_alimentar.pdf

21. Organização Mundial da Saúde (OMS). Physical status: the use and interpretation of anthropometry. Genebra: WHO; 1995.

22. Lipschitz DA. Screening for nutritional status in the elderly. Primary Care. 1994; 21(1):55-67.

23. Ministério da Saúde. Dados Consolidados do Resultado de Acompanhamento de Condicionalidades da Saúde - 1a/2019 [Internet]. Resultado de Acompanhamento de Condicionalidades da Saúde. 2019 [cited 2019 Nov 3]. Available from: https://bfa.saude.gov.br/ relatorio

24. Monteiro CA, Levy RB, Claro RM, De Castro IRR, Cannon G. Increasing consumption of ultra-processed foods and likely impact on human health: Evidence from Brazil. Public Health Nutr. 2011 Jan;14(1):5-13.

25. Brasil. Ministério da Saúde. Secretaria de Atenção Primária à Saúde. Departamento de Promoção da Saúde. Guia alimentar para crianças brasileiras menores de 2 anos. 1st ed. Ministério da Saúde, editor. Brasília; 2019. 256 p.

26. Brasil. Ministério da Saúde. Secretaria de Atenção à Saúde. Departamento de Atenção Básica. Guia alimentar para a população Brasileira [Internet]. 2nd ed. Ministério da Saúde, editor. Brasília; 2014 [cited 2020 Jan 22]. 156 p. Available from: www.saude.gov. $\mathrm{br} / \mathrm{bvs}$

27. Ferreira VR, Sangalli CN, Leffa PS, Rauber F, Vitolo MR. The impact of a primary health care intervention on infant feeding practices: a cluster randomised controlled trial in Brazil. J Hum Nutr Diet. 2019 Feb 1;32(1):21-30.

28. Vitolo MR, Louzada ML da C, Rauber F. Atualização sobre alimentação da criança para profissionais de saúde: Estudo de campo randomizado por conglomerados. Rev Bras Epidemiol. 2014 Oct 1;17(4):873-86.

29. Ministério da Saúde. Portaria no 1.920, de 5 de setembro de 2013 - Institui a Estratégia Nacional para Promoção do Aleitamento Materno e Alimentação Complementar Saudável no Sistema Único de Saúde (SUS) -Estratégia Amamenta e Alimenta Brasil. 2013.

30. Venancio SI, Giugliani ERJ, de Oliveira Silva OL, Stefanello J, Benicio MHD, Guerreiro dos Reis MC, et al. Associação entre o grau de implantação da Rede Amamenta Brasil e indicadores de amamentação. Cad Saude Publica. 2016;32(3). 
31. Passanha A, Benício MHDA, Venâncio SI, Dos Reis MCG. Implementation of the Brazilian breastfeeding network and prevalence of exclusive breastfeeding. Rev Saude Publica. 2013 Dec;47(6):1141-8.

32. Brasil. Decreto $n^{\circ} 6.286$, de 5 de dezembro de 2007. Institui o Programa Saúde na Escola - PSE, e dá outras providências. 2007.

33. Ministério da Saúde. Portaria ${ }^{\circ}$ 2.264, de 30 de agosto de 2019 Define Municípios com adesão aos Programas Saúde na Escola (PSE) e Crescer Saudável para o ciclo 2019/2020, os habilita ao recebimento do teto de recursos financeiros pactuados em Termo de Compromisso e dá outr. 2019.

34. Cardoso MA, Augusto RA, Bortolini GA, Oliveira CSM, Tietzman DC, Sequeira LAS, et al. Effect of providing multiple micronutrients in powder through primary healthcare on anemia in young brazilian children: A multicentre pragmatic controlled trial. PLoS One. 2016 Mar 1;11(3)

35. Ministério da Saúde. Portaria ${ }^{\circ} 424$, de 19 de março de 2013 -Redefine as diretrizes para a organização da prevenção e do tratamento do sobrepeso e obesidade como linha de cuidado prioritária da Rede de Atenção à Saúde das Pessoas com Doenças Crônicas. 2013.

36. Brasil. Instituto Brasileiro de Geografia e Estatística. Pesquisa nacional de saúde : 2013 : percepção do estado de saúde, estilos de vida e doenças crônicas: Brasil, grandes regiões e unidades da federação. Rio de Janeiro: IBGE, Coordenação de Trabalho e Rendimento; 2014. 181 p.

37. Sharma AM, Bélanger A, Carson V, Krah J, Langlois M, Lawlor D, et al. Perceptions of barriers to effective obesity management in Canada: Results from the ACTION study. Clin Obes. 2019;9(5):1-11.

Manuscrito recebido em 20 de novembro de 2019. Aceito em versão revisada em 4 de fevereiro de 2020

\section{Feeding and nutrition efforts in the context of primary healthcare in Brazil}

ABSTRACT In Brazil, the promotion, protection, and recovery of health are among the initiatives provided by the public and universal Unified Health System (SUS). The primary healthcare level (PHC), the preferred point of access to the system, plays the role of coordinating and ordinating health care services and actions in the network. In the context of PHC, feeding and nutrition efforts must be aligned with National Feeding and Nutrition Policy (PNAN) guidelines and may be supported by feeding and nutritional surveillance actions. Data from PHC information systems and population surveys show that excess weight affects more than half the adult population of Brazil, and that consumption of ultra-processed foods is on the rise. This scenario requires that health care teams be prepared to prioritize initiatives for users with chronic diseases, using risk stratification, stabilization of the disease, and enhancement of supported self-care with a focus on diet and physical activity. At the same time, considering the country's epidemiological profile, teams must carry out initiatives to fight undernutrition and prevent anemia and hypovitaminosis A to address the multiple burden of malnutrition. The present article describes the current scenario of feeding and nutrition initiatives implemented at the PHC level in Brazil via the SUS.

Keywords $\quad$ Public policy; obesity; diabetes mellitus; hypertension; nutrition programs and policies; Brazil.

\section{Medidas relativas a la alimentación y la nutrición en la atención primaria de salud en Brasil}

RESUMEN En Brasil, el Sistema Único de Salud (SUS), que es público y universal, ofrece medidas de promoción, protección y recuperación de la salud. La atención primaria de salud es la puerta preferida de acceso de la población al SUS, y su función es coordinar y ordenar las medidas y los servicios de salud disponibles en la red. En el ámbito de la atención primaria de salud, las medidas de alimentación y nutrición deben estar en consonancia con las directrices de la política nacional de alimentación y nutrición, y pueden potenciarse a partir de medidas de vigilancia alimentaria y nutricional. Los datos de los sistemas de información disponibles en todas las unidades básicas de salud y en encuestas poblacionales indican que más de la mitad de la población adulta en Brasil presenta exceso de peso y que ha aumentado el consumo de alimentos ultraprocesados. Esa situación exige que los equipos de salud se organicen con objeto de priorizar ciertas medidas para las personas con trastornos crónicos, según la estratificación del riesgo, la estabilización del trastorno y la potenciación del autocuidado apoyado, con enfoque en la alimentación y la actividad física. Al mismo tiempo, según el perfil epidemiológico, los equipos deben emprender actividades de lucha contra la desnutrición y de prevención de la anemia y de la carencia de vitamina A, teniendo en cuenta la múltiple carga de la malnutrición en el país. El objetivo del presente artículo es presentar el panorama actual de las medidas relativas a la alimentación y nutrición que se llevan a cabo en el ámbito de la atención primaria de salud en el SUS.

Palabras clave Política pública; obesidad; diabetes mellitus; hipertensión; programas y políticas de nutrición y alimentación; Brasil. 